\title{
Transcatheter closure of ventricular septal defects with nitinol wire occluders of type patent ductus arteriosus
}

\author{
Arkadiusz Wierzyk ${ }^{1}$, Małgorzata Szkutnik ${ }^{1}$, Roland Fiszer ${ }^{1}$, Paweł Banaszak ${ }^{1}$, Szymon Pawlak², \\ Jacek Białkowski ${ }^{1}$ \\ ${ }^{1}$ Clinical Ward of Congenital Heart Diseases and Pediatric Cardiology in Zabrze, Silesian Medical University, Silesian Centre for Heart \\ Diseases, Zabrze, Poland \\ ${ }^{2}$ Chair and Clinical Department of Cardiac Surgery and Transplantology in Zabrze, Silesian Medical University, Silesian Centre for Heart \\ Diseases, Zabrze, Poland
}

Postep Kardiol Inter 2014; 10, 1 (35): 21-25

DOI: $10.5114 /$ pwki.2014.41462

\begin{abstract}
A bstract
Ventricular septal defects closure (VSD) depending on the anatomy and clinical setting can be performed surgically or by a hybrid and transcatheter approach. Two cases of children with VSD will be presented. Patients' defects were closed with various types of occluders made of nitinol wire mesh occluder, patent ductus arteriosus (PDA) type. The first case was a 2.5-year-old boy after cardiosurgical correction of tetralogy of Fallot (TOF). After the procedure, a significant haemodynamic residual VSD was observed, which was not successfully closed during the subsequent reoperation. Despite pharmacological treatment, symptoms of heart failure were observed in this patient. In echocardiographic images the residual VSD was presented as a tunnel-like dissection of the ventricular septum (length $6 \mathrm{~mm}$ and diameter $3.4 \mathrm{~mm}$ ). The defect was closed via arterial access with an Amplatzer Duct Occluder II (ADO II). The procedure was successfully performed without any medical complications. In this child, a significant shunt reduction and a noticeable improvement in the patient's clinical status and diminished symptoms of heart failure were noticed. The second patient was a 4-year-old girl suffering from a multi-perforated perimembranous VSD accompanied by a ventricular septal defect with aneurysm. The defect was closed by a venous approach with a PDA Cardio-O-Fix occluder (very similar to ADO I). No short-term or long-term complications were visible during or after the procedure. Only a mild residual shunt through the VSD was observed 6 months afterwards. Transcatheter VSD closure with a proper morphology, with occluders of type Amplatzer Duct Occluder ADO I or ADO II, constitutes a safe and effective therapeutic alternative.
\end{abstract}

Key words: transcatheter closure, ventricular septal defect, congenital heart defect.

\section{Introduction}

Hemodynamically significant ventricular septal defects closure (VSD), isolated as well as being an integral part of complex congenital heart defects, are closed surgically in the majority of cases. Notwithstanding, there is a group of patients in whom a transcatheter closure brings potentially greater benefits to patients than surgery (the less invasive the method of the procedure, the lower the risk of adverse reactions). It pertains to defects with specific morphology such as muscular ventricular septal defects (mVSD) and residual ventricular septal defects (re-VSD) occurring after previous surgical treatment $[1,2]$. Implants, which are generally applied for that reason, are special VSD devices.
We present here two examples of VSD transcatheter closure - one residual postsurgery ventricular septal defect (re-VSD) and the second native perimembranous VSD (pmVSD) accompanied by intraventricular septal (IVS) aneurysm. Both of them were closed with different types of occluders made of nitinol wire mesh, which were originally designed for closure of large patent ductus arteriosus (PDA).

\section{Case reports}

\section{Case 1}

A 2.5-year-old boy underwent two cardiosurgical corrections of tetralogy of Fallot (TOF) at the age of 2 and was reoperated on 5 months afterwards due to re-VSD.

\section{Corresponding author:}

Arkadiusz Wierzyk MD, Clinical Ward of Congenital Heart Diseases and Pediatric Cardiology in Zabrze, Silesian Medical University, Silesian Centre for Heart Diseases, 9 M. Curie-Skłodowskiej St, 41-800 Zabrze, Poland, phone: +48 601 618 336, e-mail: awierzyk@mp.pl Received: 16.01.2014, accepted: 17.01.2014. 
Just after the second procedure, transesophageal echocardiography (TEE) showed total closure of the re-VSD. However, during the following transthoracic echocardiography (TTE), conducted after moving the patient from an intensive care unit, the defect in the place of a patch size 4-5 $\mathrm{mm}$ reappeared. Pharmacological treatment was applied, namely captopril, furosemide, spironolactone as well as digoxin. During ambulatory observation incremental signs and symptoms of heart failure (HF) were diagnosed and the boy was admitted to the hospital again. Symptoms of poor growth were sustained (body mass $13 \mathrm{~kg}-25^{\text {th }}$ centile), tachypnea $30-40$ breaths. A clinical
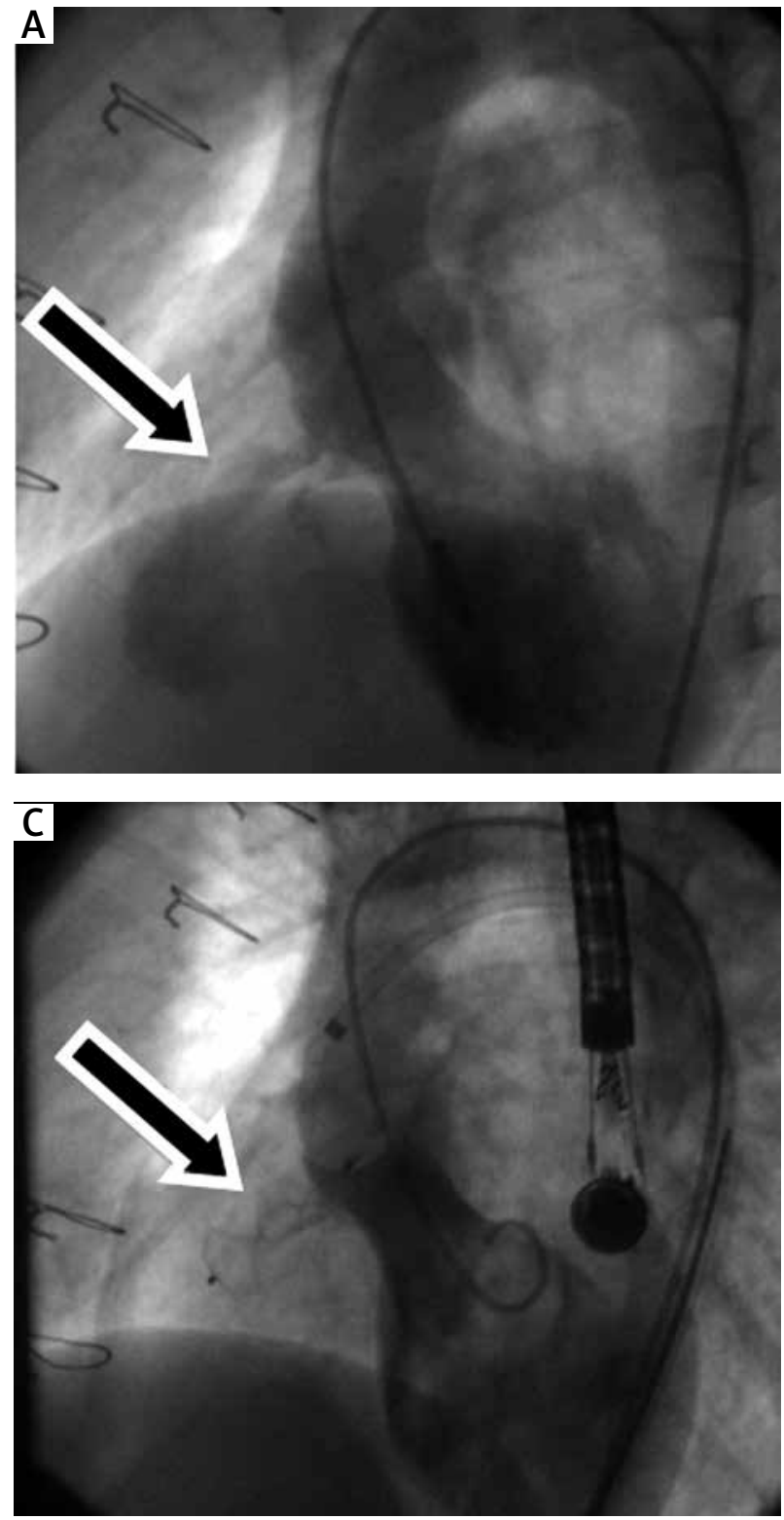

examination showed: precordial thrill, systolic murmur at left sternal side $4 / 6$ according to Levin, hepatomegaly approximately $2 \mathrm{~cm}$ below the right rib cage. Saturation was $92-94 \%$. Thorax radiography showed significantly increased vascularity of the lungs. Electrocardiography (ECG) indicated characteristics of right bundle branch block (RBBB) and left anterior hemiblock (LAH). In UCG enlarged right ventricle (RV) and left atrium were seen. The residual VSD was presented as a 'tunnel- like' dissection of the ventricular septum, length $6 \mathrm{~mm}$ and diameter $3.4 \mathrm{~mm}$. The patient's parents did not agree to further surgical intervention. Considering that fact, the decision
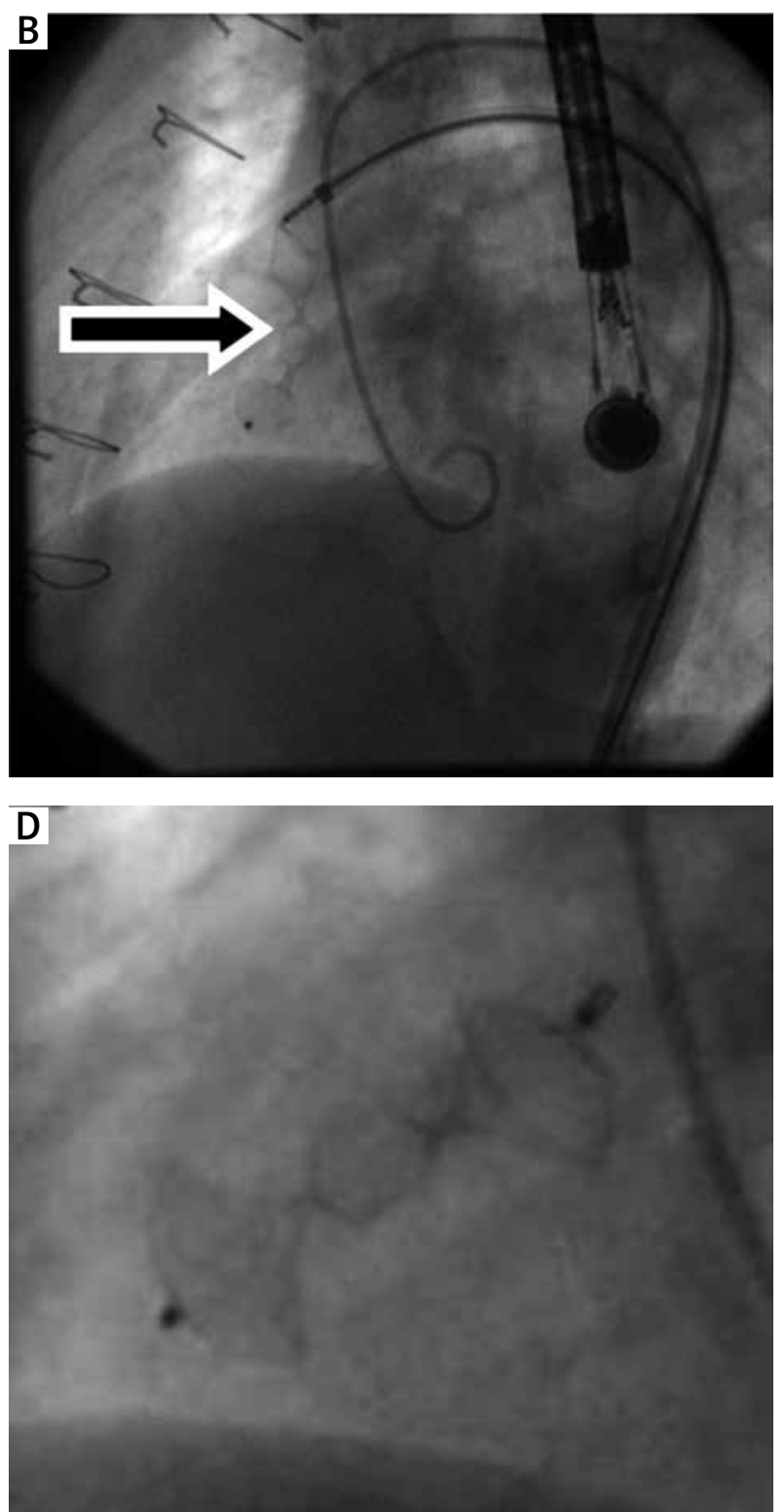

Figure 1. Residual ventricular septal defect closure with ADO II. LAO 30 projection. A - Left ventriculography before the closure, visible 'tunnel-like' VSD. B - Occluder still connected to the delivery system (arrow). C - Left ventriculography showing the position of the occluder - waist of ADO II fills in canal type VSD (arrow). D - Occluder released from the delivery system (fluoroscopy magnification) 
of an attempt at transcatheter closure of the re-VSD was made.

Re-VSD closure was done under general anesthesia, with antibiotic protection (cefazolin $100 \mathrm{mg} / \mathrm{kg}$ body weight, administered intravenously) and under TEE control. Before the procedure, one dose of heparin $50 \mathrm{U} / \mathrm{kg}$ body weight was administered intravenously. The right artery and femoral vein were cannulated. Thereafter, $4 \mathrm{Fr}$ sheaths and diagnostic catheters were implemented. Invasive measurement of blood pressure was performed. Findings were as follows: in RA - 30/22/26, in $\mathrm{RV}-70 / 0 / 22$, in $\mathrm{PA}-48 / 23 / 35$, in $\mathrm{LV}-125 / 0 / 22$ and in Ao $-113 / 68 / 92 \mathrm{~mm} \mathrm{Hg}$. The size of shunt was measured (Qp : Qs 1.5 : 1) as well as resistance (PVR 5 Wood units, SVR 21.4 units). Left ventriculography showing re-VSD was carried out (Figure 1 A). Thereafter, a JR 5 Fr cathe-

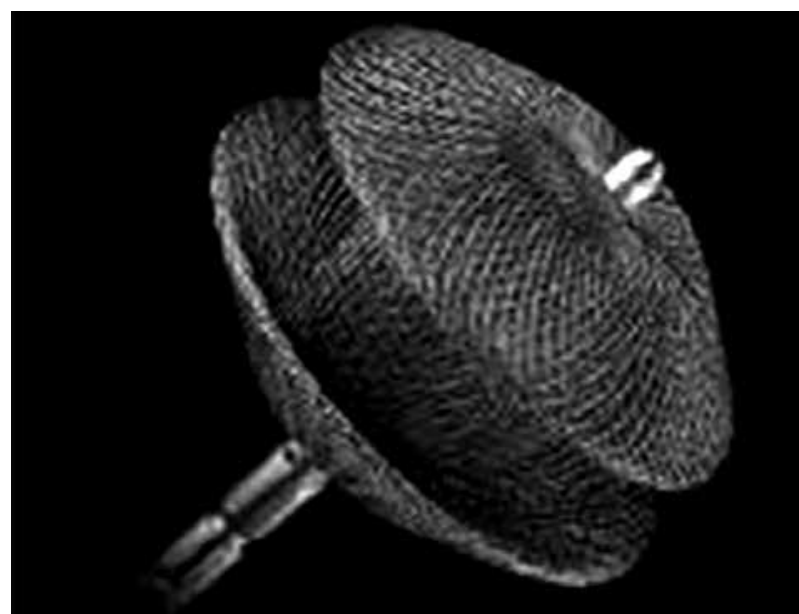

Figure 2. Amplatzer Duct Occluder type II (ADO II)
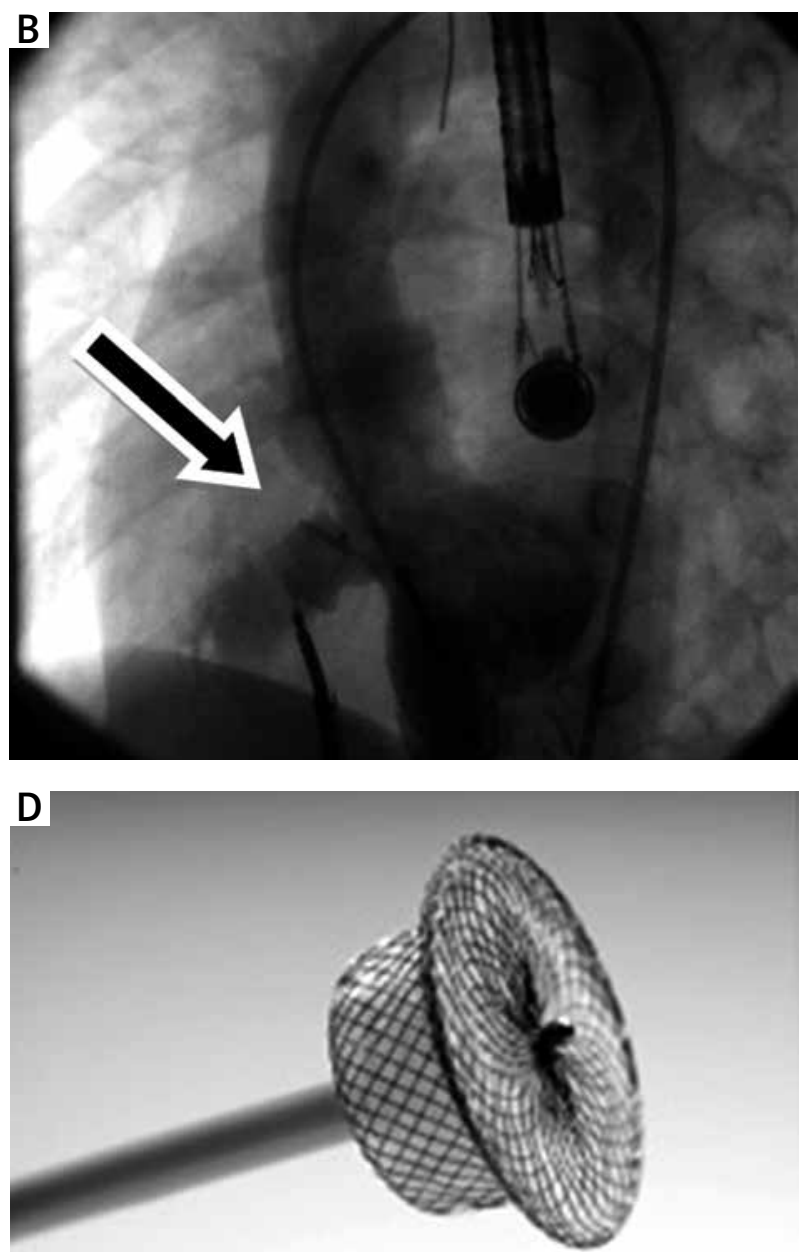

Figure 3. Perimembranous VSD closure with aneurysm with occluder type COF PDA. Left ventriculography. LAO 30 projection. A - VSD before closure. B - Occluder in the diameter of the aneurysm, still connected to the delivery system. C-After closure of the defect. D - Cardi-O-Fix PDA Occluder 
ter was implemented with a hydrophilic guidewire from the left ventricle, through the re-VSD, to the right ventricle. The JR catheter was exchanged for a $5 \mathrm{Fr}$ sheath introducer and a size $6 / 6 \mathrm{~mm}$ Amplatzer Duct Occluder type II - ADO II (AGA Med. Corp., USA - Figure 2) was implemented through that sheath. The aim was to open a distal disk in the RV and a proximal disk in the left ventricle. In order to achieve that, it was necessary to conduct a twofold reposition of the occluder (Figures $1 \mathrm{~B}-\mathrm{D}$ ). The total duration of the procedure was $2 \mathrm{~h}$, including $16.8 \mathrm{~min}$ of fluoroscopy. After the procedure, left ventriculography was carried out. A mild residual shunt without hemodynamic significance was visible. In TEE the correct position of the ADO II was confirmed. Trivial aortic valve regurgitation was diagnosed.

The boy was rehospitalized 3 months after the procedure. Significantly diminished symptoms of HF were observed in this patient. The TTE showed a mild shunt in the occluder area, hemodynamically insignificant. The patient's body mass was then $15.7 \mathrm{~kg}\left(25^{\text {th }}-50^{\text {th }}\right.$ centile). During the period of hospitalization, cardiac rhythm abnormalities (which had been diagnosed previously) were noticed a supraventricular tachycardia and one episode of bradycardia with decrease in heart rate to 38 beats per minute (bpm) in Holter ECG. Because of the resistance of supraventricular tachycardia to pharmacological treatment (no response to adenosine) the attack was interrupted by cardioversion. Thereafter, ablation RF with a satisfactory result was conducted. At preset, one year after the procedure, the patient feels well and he is under ambulatory care.

\section{Case 2}

A 4-year-old girl with congenital heart defect - native perimembranous VSD partially covered by IVS aneurysm - was admitted to hospital in order to carry out a VSD transcatheter closure. Congenital heart defect was diagnosed just after the delivery. From that time, the girl was under the Pediatric Cardiac Department's care. Pharmacological treatment was not applied to the patient.

After admission to the hospital a clinical examination was carried out. The examination included the following aspects: holosystolic murmur at left sternal side 3-4/6 of Levin scale and a mild parasternal thrill. The patient's body mass was $13 \mathrm{~kg}$ ( $25^{\text {th }}$ centile). Thorax radiography showed slightly increased vascularity as well as enlarged heart. The TTE examination showed at least 2 defects approximately $2 \mathrm{~mm}$ each in the perimembranous IVS with aneurysm size $8 \mathrm{~mm}$, left-to-right shunt, gradient about $90 \mathrm{~mm} \mathrm{Hg}$.

Cardiac catheterization was done under general anesthesia and with antibiotic protection; $50 \mathrm{U} / \mathrm{kg}$ body weight of heparin was administered. The right artery and femoral vein were cannulated. The measurement of blood pressure was checked; findings were as follows: RA - 11/9/9, RV - 38/0/11, PA - 34/16/24, LV - 110/0/20 and Ao $-100 / 61 / 81 \mathrm{~mm} \mathrm{Hg}$. Qp/Qs was 1.6. During the left ventriculography, the aneurysm (diameter approximately $8 \mathrm{~mm}$ ) in the perimembranous IVS with at least 2 defects ( $2 \mathrm{~mm}$ each) was revealed (Figure $3 \mathrm{~A}$ ). After the removal of diagnostic catheters, a catheter type JR $5 \mathrm{Fr}$ was implemented from the LV through the VSD, to the RV. Thereafter, a guidewire $(0.035 \mathrm{~cm} \times 260 \mathrm{~cm})$ was inserted into the left pulmonary artery and a $15 \mathrm{~mm}$ Andrasnare was introduced from the femoral vein to the left pulmonary artery and an arterio-venous loop was created. A transseptal sheath (180 st. 7 Fr) was implemented through the vein using the 'kissing method'. After placing the sheath into an ascending aorta, the dilator was removed. A Cardi-O-Fix PDA Occluder (COF PDA - Starway Medical Technology Inc., China - Figure 3 D) size $8 / 6 \mathrm{~mm}$ was inserted. Having released the retentional ventricular disk, the whole delivery system was withdrawn to the IVS aneurysm. Thereafter, a proximal part of the device was opened (Figures 3 B and $3 \mathrm{C}$ ). Occluder implantation was monitored by TEE and fluoroscopy as well. The total duration of the procedure was $2.5 \mathrm{~h}$, including $23.4 \mathrm{~min}$ of fluoroscopy. During the examination, carried out 6 months after the implantation of the occluder, TTE diagnosed residual shunt in the lower and upper pole of the occluder. The patient felt well and pharmacological treatment was not applied. The patient's weight was $20 \mathrm{~kg}$ ( $90^{\text {th }}$ centile), height $113 \mathrm{~cm}$ (90 9 th $^{\text {ch }}$ centile). During the clinical examination a mild murmur in the left sternal side was heard without other symptoms. No heart rhythm or conduction disorders were diagnosed (also in Holter EKG).

\section{Discussion}

The cases presented here indicate the utility of applied PDA nitinol occluders in transcatheter closure of selected types of VSD. The frequency of residual, post-surgical VSD constitutes $5-25 \%$. Residual VSD may be the consequence of an unstitched patch or leak closure. A small re-VSD (diameter $<2 \mathrm{~mm}$ ) is usually closed spontaneously [1-4]. Bigger re-VSD frequently cause significant hemodynamic consequences and the necessity of more complicated reoperation. Transcatheter closure of this defect is an alternative for patients.

Based on the literature, asymmetric and symmetric Amplatzer Ventricular Septal Occluders [5, 6] or modified Chinese occluders [7] were successfully used for percutaneous closure of re-VSD. After insertion, the most severe complications were intravenous hemolysis (after implantation) or cardiac rhythm abnormalities diagnosed during or after the procedure [5-7]. In a case similar to that presented here (re-VSD after TOF correction) also a different device, a mushroom-shaped Amplatzer Duct Occluder type I (ADO I), was used successfully (Figure 3 D) [8].

There are not many articles in the literature describing the usage of Amplatzer Duct Occluder type II (ADO II) for VSD closure. It mainly concerns native VSD. In 2012 Koneti et al. [9] described transcatheter closure of perimembra- 
nous VSD with ADO II in 57 children (aged $0.7-14.5$ years old). Size of the defect was 3.4-6.5 $\mathrm{mm}$ (average $5 \mathrm{~mm}$ ). The effectiveness of the procedure was $94 \%$ (permanent closure of the defect) and remote consequences were estimated as promising. In the case of one patient, embolization of the occluder occurred. The described study case indicates beneficial and highly effective usage of ADO II in a child suffering from recurrent, post-surgical re-VSD after previous correction of entire TOF.

Our experience with percutaneous closure of native perimembranous VSD with IVS aneurysm by Duct Occluder I is consistent with a report published recently by El Said et al. [10]. The authors of that article described a group of 21 patients (age 15 months to 30 years) with a similar VSD morphology treated with ADO I. In their study before the procedure, TTE showed a defect diameter in the range $2.5-8 \mathrm{~mm}$ (average $5 \mathrm{~mm}$ ) and aneurysm 8.4-16 mm (average $11 \mathrm{~mm}$ ). The procedure was carried out on 19 patients; 2 patients were withdrawn from the procedure. In 1 patient, while inserting the catheter through the defect, symptoms of total atrioventricular block (TAVB) appeared. Ventricular tachycardia occurred in the second patient. As far as our case is concerned, no complications were observed during and after the procedure. After the implantation residual shunt (hemodynamically insignificant) was observed, which also occurred in 7 out of 19 patients described in the publication written by El Said et al. [10]. This may suggest that more precise selection of patients who require such interventions is required. The usage of two overlapping ADO II can be an interesting alternative for VSD with multiperforated aneurysm of the intraventricular septum [11].

In transcatheter VSD closure, ADO I and ADO II are used in order to eliminate the risk of total atrioventricular block (TAVB) observed in follow-up with the use of the Amplatzer Perimembranous Ventricular Septal Occluder [12, 13]. Application of this device in children below 3 years old can lead to that complication in up to $18 \%$ of patients [14]. Theoretically, lower risk of TAVB is connected with a gentle construction of ADO II, namely thinner structure of the nitinol wire and lack of patches inside the occluder. In the case of $A D O$ I there is a lack of a right retentional disk. In follow-up the appearance of TAVB, after the use of ADO I or ADO II for VSD closure, has not been described in any available literature (similarly to our study cases).

An additional interesting aspect of this article is showing that Chinese PDA devices (very similar to ADO I) are as effective as original occluders. We documented that fact also in cases of transcatheter closure of ruptured sinus of Valsalva aneurysm [15] and coronary fistulas $[16,17]$.

\section{Conclusions}

Transcatheter VSD closure with a proper morphology, with occluders of type Amplatzer Duct Occluder, consti- tutes a safe and effective therapeutic alternative in selected patients.

\section{References}

1. Hassan MK, Hasan KA, Rahim AMA, et al. Residual ventricular septal defect after surgical repair of TOF - a case report. Cardiovasc J 2011; 3: 233-234.

2. Erdem S, Ozbarlas N, Küçükosmanoglu O, et al. Long term follow-up of 799 children with isolated ventricular septal defects. Turk Kardiyol Dern Ars 2012; 40: 22-25.

3. Miyake T, Shinohara T, Nakamura Y, et al. Spontaneous closure of ventricular septal defects followed up from <3 months of age. Pediatr Int 2004; 46: 135-140.

4. Dodge-Khatami A, Knirsch W, Tomaske M, et al. Spontaneous closure of small residual ventricular septal defects after surgical repair. Ann Thorac Surg 2007; 83: 902-905.

5. Walsh MA, Coleman DM, Oslizlok P, et al. Percutaneous closure of postoperative ventricular septal defects with the Amplatzer device. Catheter Cardiovasc Interv 2006; 67: 445-451.

6. Dua JS, Carminati M, Lucente M, et al. Transcatheter closure of postsurgical residual ventricular septal defects: early and midterm results. Catheter Cardiovasc Interv 2010; 75: 246-255.

7. Gu MB, Bai Y, Zhao XX, et al. Transcatheter closure of postoperative residual perimembranous ventricular septal defects. Ann Thorac Surg 2009; 88: 1551-1555.

8. Vaidyanathan B, Kannan BR, Kumar RK. Device closure of residual ventricular septal defect after repair of tetralogy of Fallot using the amplatzer duct occluder. Indian Heart J 2005; 57: 164-166.

9. Koneti NR, Sreeram N, Penumatsa RR, et al. Transcatheter retrograde closure of perimembranous ventricular septal defects in children with the Amplatzer duct occluder II device. J Am Coll Cardiol 2012; 60: 2421-2422.

10. El Said HG, Bratincsak A, Gordon BM, et al. Closure of perimembranous ventricular septal defects with aneurysmal tissue using the Amplazter Duct Occluder I: lessons learned and medium term follow up. Catheter Cardiovasc Interv 2012; 80: 895-903.

11. Devendran V, Koneti NR, Jesudian V. Transcatheter closure of multiple perimembranous ventricular septal defects with septal aneurysm using two overlapping Amplatzerr Duct Occluders II. Pediatr Cardiol 2013; 34: 1963-1965.

12. Walsh MA, Bialkowski J, Szkutnik M, et al. Atrioventricular block after transcatheter closure of perimembranous ventricular septal defects. Heart 2006; 92: 1295-1297.

13. Zhou T, Shen XQ, Zhou SH, et al. Atrioventricular block: a serious complication in and after transcatheter closure of perimembranous ventricular septal defects. Clin Cardiol 2008; 31: 368-371.

14. Predescu D, Chaturvedi RR, Friedberg MK, et al. Complete heart block associated with device closure of perimembranous ventricular septal defects. J Thorac Cardiovasc Surg 2008; 136: 1223-1228.

15. Bialkowski J, Akdeniz C, Celebi A. Transcatheter closure in two rare cases of left-to-right shunt with Cardio-O-Fix occluders. Cardiol Young 2012; 22: 96-99.

16. Białkowski J, Szkutnik M, Fiszer R, Zembala M. Transcatheter occlusion of a large coronary artery fistula using a patent ductus arteriosus occluder. Kardiol Pol 2011; 69: 1318-1319.

17. Białkowski J, Szkutnik M, Zhang G, Jiang S. Large coronary artery fistula and patent ductus arteriosus: transcatheter closure with three PDA nitinol wire mesh occluders. Postep Kardiol Inter 2013; 9: 89-92. 